\title{
Validade do ergômetro de braço para a determinação do limiar anaeróbio e da performance aeróbia de nadadores
}

\section{LGA Guglielmo}

Faculdades Salesianas de Lins

Laboratório de Avaliação do Esforço Físico

Faculdade de Educação Física

Lins, São Paulo

Brasil

https://doi.org/10.5628/rpcd.01.03.07

\author{
BS Denadai
}

Laboratório de Avaliação da Performance Humana

UNESP

Rio Claro, São Paulo

Brasil

\section{RESUMO}

O objetivo deste estudo foi analisar a validade da intensidade correspondente ao limiar anaeróbio (LAn) e à potência máxima (Pmax), obtida durante um teste incremental em um ergômetro específico de braço, predizer o desempenho na distância de $400 \mathrm{~m}$ e a intensidade do LAn determinados na natação (P). Participaram do estudo 16 nadadores aparentemente saudáveis do sexo masculino. Todos os nadadores foram submetidos aos seguintes testes: 1) 1 x $400 \mathrm{~m}$ no estilo crawl em intensidade máxima; 2) 3 x $400 \mathrm{~m}$ a uma intensidade de $80 \%$, $85 \%$, e $90 \%$ do seu melhor tempo para a distância com objetivo de determinar o LAn e; 3) teste incremental e contínuo em um ergômetro do braço (EB) para a determinação da intensidade correspondente ao LAn. A Pmax expressa em $\mathrm{W}$ e em W/kg não foi significantemente correlacionada com a velocidade do LAn $(\mathrm{r}=$ 0,37 e $r=0,29$ ) e foi significantemente correlacionada com o melhor tempo para nadar os $400 \mathrm{~m}(\mathrm{r}=-0,66 \mathrm{e} \mathrm{r}=-0,57)$. A velocidade correspondente ao LAn no protocolo de $\mathrm{P}$ foi significantemente correlacionada $(r=0,75)$ com a carga do LAn no EB. O melhor tempo para nadar os $400 \mathrm{~m}$ foi significantemente correlacionado com a carga do LAn no EB e a velocidade do LAn no protocolo de $\mathrm{P}(\mathrm{r}=-0,74 ; \mathrm{r}=-0,90$, respectivamente). Pode-se concluir que a validade do ergômetro de braço para a avaliação aeróbia de nadadores é mais elevada quando a resposta do lactato sanguíneo (LAn) é utilizada ao invés dos valores de Pmax obtidos durante um teste incremental.

Palavras-chave: ergômetro de braço, natação, limiar anaeróbio, especificidade.

\author{
ABSTRACT \\ Validity of Arm Ergometer to determine the Anaerobic Threshold \\ and the Aerobic Performance of Swimmers
}

The aim of this study was to analyse the validity of the intensity corresponding to the anaerobic threshold (AT) and the maximum power (Pmax) obtained during an incremental test determined on a specific arm ergometer to predict the performance in a $400 \mathrm{~m}$ crawl sprint and intensity of the AT determined in the swimming pool $(S)$. The experiment was conducted with 16 healthy male volunteers swimmers. All subjects were submitted to the following tests: 1) a crawl sprint of 400 $\mathrm{m}$; 2) 3 crawl sprints of $400 \mathrm{~m}$ at an intensity of $80 \%, 85 \%$, and $90 \%$ of their best velocity at each one of the distances in order to determine the AT and 3) a progressive continuous test on an arm ergometer (AE) for the determination of an intensity corresponding to the AT. The Pmax expressed in $W$ and $W / \mathrm{kg}$ were not significantly correlated with the velocity of the AT $(r=0.37$ and $r=0.29)$ and significantly correlated with the best time for the $400 \mathrm{~m}$ crawl $(r=-0.66$ and $r=$ -0.57). The velocity corresponding to the AT in $S$ protocol was significantly correlated $(r=0.75)$ with the load referring to the AT during the AE protocol. The shortest time taken to complete the $400 \mathrm{~m}$ crawl was significantly correlated $(r=-0.74 ; r=-0.90)$ with the load referring to the $A T$ during the $A E$ protocol and the velocity referring to the AT in the $S$ protocol, respectively. It can be concluded that the validity of the arm ergometer for aerobic evaluation of swimmers is higher when the blood lactate response (AT) is used rather than the values of Pmax obtained during an incremental test.

Keywords: arm ergometer, swimming, anaerobic threshold, specificity. 


\section{INTRODUÇÃO}

Um grande número de pesquisadores tem procurado analisar a validade dos testes que são empregados para a avaliação aeróbia e anaeróbia de nadadores, utilizando para isso diferentes índices fisiológicos, como o consumo máximo de oxigênio (VO2max), resposta do lactato sanguíneo ao exercício, velocidade crítica e as potências pico e média obtidas durante o teste de Wingate $(9,11,20,27)$. Nestes estudos, o princípio da especificidade tem sido um dos aspectos mais investigados, com a maioria dos resultados indicando a necessidade das avaliações dos nadadores serem realizadas em um ergômetro específico (swimming flume) ou na própria piscina $(4,20)$. Como isso nem sempre é possível, alguns estudos têm procurado analisar a validade do ergômetro de braço para a avaliação aeróbia $(12,17,21)$ e anaeróbia de nadadores $(2,9,11,12)$. Especificamente em relação a avaliação aeróbia, o único índice analisado até o momento foi o $\mathrm{VO}_{2} \max$, com as informações ainda sendo contraditórias. Obert et al. (21) encontram correlação significante entre o $\mathrm{VO}_{2}$ max obtido no ergômetro de braço e a performance na natação. Por outro lado, Loftin et al. (17) não encontraram correlação significante entre a performance de triatletas na natação e o $\mathrm{VO}_{2}$ max determinado no ergômetro de braço.

Em princípio estes dados contraditórios não podem ser explicados apenas pela possível falta de especificidade do ergômetro de braço, pois o $\mathrm{VO}_{2}$ max também pode não ser o melhor índice para a predição de performance aeróbia, mesmo quando a avaliação é feita no swimming flume (27), no tethered swimming (14) ou na piscina $(5,28)$. Por outro lado, muitos estudos utilizando diferentes tipos de exercício (6, $27,29)$ têm verificado que os índices associados a resposta do lactato sanguíneo (limiar de lactato, limiar anaeróbio, início do acúmulo de lactato no sangue, máxima fase estável de lactato) são melhores preditores da performance aeróbia em exercícios de média ou longa duração (acima de 10 minutos) do que o $\mathrm{VO}_{2}$ max.

Outro aspecto que pode explicar em parte estes resultados discordantes é a utilização, em alguns destes estudos, de um ergômetro de braço que foi adaptado para a avaliação dos atletas. Estudos prévios têm verificado que as dimensões do ergômetro e consequentemente a postura adotada durante o teste, podem influenciar as respostas fisiológicas durante o exercício realizado no ergômetro de braço $(24,26)$.

Com base nestas informações, o objetivo deste estudo foi analisar a validade da intensidade correspondente ao limiar anaeróbio (LAn) e da potência máxima (Pmax) obtida durante um teste incremental determinado em um ergômetro de braço específico, para predizer a performance na prova de $400 \mathrm{~m}$ nado crawl e a intensidade do LAn determinado na piscina.

\section{MATERIAL E MÉTODOS \\ Condições ambientais}

Os testes no ergômetro de braço foram realizados em um laboratório, com a temperatura ambiente variando entre $22-24^{\circ} \mathrm{C}$ e a umidade relativa do ar entre $60-70 \%$. Os testes de campo foram realizados em uma piscina de $25 \mathrm{~m}$ com a temperatura da água variando entre $25-27^{\circ} \mathrm{C}$.

\section{Sujeitos}

Participaram deste estudo 16 voluntários aparentemente saudáveis do sexo masculino. Todos os sujeitos eram treinados, sendo 13 nadadores e 3 triatletas. Todos os atletas encontram-se seriamente envolvidos em seus programas de treinamento, no mínimo há dois anos. Os nadadores e triatletas realizavam um treinamento de natação de $6 \mathrm{~km} /$ dia; 6 dias/semana e de $3 \mathrm{~km} / \mathrm{dia} ; 4$ dias/semana, respectivamente. Todos os sujeitos assinaram um termo de consentimento, tendo o experimento sido aprovado pelo Comitê de Ética em Pesquisa do IB/UNESP Rio Claro. As características dos nadadores e a performance na prova de $400 \mathrm{~m}$ nado crawl estão expressos na Tabela 1.

Tabela 1 - Características físicas e de performance dos nadadores ( $N=16$ ).

\begin{tabular}{lccccc} 
& $\begin{array}{c}\text { Idade } \\
\text { (anos) }\end{array}$ & $\begin{array}{c}\text { Massa } \\
\text { corp. (kg) }\end{array}$ & $\begin{array}{c}\text { Altura } \\
\text { (cm) }\end{array}$ & $\begin{array}{c}\text { Gordura } \\
\text { corp. (\%) }\end{array}$ & $\begin{array}{c}\text { T 400m } \\
\text { (s) }\end{array}$ \\
\hline Média & 18,4 & 67,3 & 170,0 & 17,6 & 320,2 \\
DP & 2,5 & 6,9 & 5,0 & 3,8 & 25,0
\end{tabular}

T 400 m - melhor tempo de 400 m nado crawl 


\section{Testes de campo}

Os sujeitos realizaram uma repetição de $400 \mathrm{~m}$ com intensidade máxima no nado $\mathrm{crawl}$, registrando-se os tempos por meio de um cronômetro manual.

Posteriormente, foram submetidos a três repetições de $400 \mathrm{~m}$ em nado crawl a uma intensidade de $80 \%$, $85 \%$ e $90 \%$, respectivamente, da sua melhor velocidade para esta distância, com 20 minutos de recuperação entre cada repetição (19). Após 1, 3 e 5 minutos de recuperação, foram coletadas $25 \mu \mathrm{l}$ de sangue do lóbulo da orelha. Para a determinação do LAn foi considerado apenas a mais alta concentração de lactato entre as três amostras de cada repetição. Deste modo, para cada repetição, foi determinada a velocidade média $(\mathrm{m} / \mathrm{s})$ e sua respectiva concentração de lactato e por interpolação linear, foi calculada a velocidade correspondente a $4 \mathrm{mM}$ de lactato (LAn). Ao final de cada $400 \mathrm{~m}$ a freqüência cardíaca (FC) foi mensurada e, do mesmo modo do que para a velocidade, foi calculada a FC equivalente a $4 \mathrm{mM}$.

\section{Teste de laboratório}

Os sujeitos foram submetidos a um teste contínuo e progressivo em um ergômetro específico de braço (UBE 2462 - Cybex), para a determinação das potências (W e W/kg) equivalentes ao LAn e a Pmax. O ergômetro foi ajustado de modo que ombro permanecesse na mesma altura do eixo da manivela, e os cotovelos não ficassem completamente estendidos, empregando-se uma rotação fixa de $60 \mathrm{rpm}$. A carga inicial foi de $33,3 \mathrm{~W}$, com incrementos de 16,6 W a cada 3 minutos, até a exaustão voluntária. Ao final de cada estágio foram mensuradas a FC (Polar-Vantage XL) e também foi coletada uma amostra de sangue $(25 \mu \mathrm{l})$ do lóbulo da orelha. O LAn foi determinado como sendo a potência e a FC equivalentes à concentração de 3,5 mM de lactato (13). Com base nos dados de Heck et al. (13), protocolos incrementais e contínuos com estágios inferiores a cinco minutos devem utilizar a concentração fixa de 3,5 $\mathrm{mM}$ de lactato ao invés de $4 \mathrm{mM}$, para não superestimar a intensidade do LAn.

A Pmax foi empregada neste estudo como um índice indireto da potência aeróbia máxima (16). A Pmax foi definida com a potência correspondente ao último estágio completo do teste incremental. Se o indivíduo não completou o estágio (menos do que 3 min), a Pmax foi determinada levando-se em consideração o tempo de permanência no último estágio, conforme a fórmula proposta por Hawley et al. (11):

$\operatorname{Pmax}(\mathrm{W})=$ Pcom $+(\mathrm{t} / 180 \times \Delta \mathrm{P})$

onde : Pmax é a potência máxima ; Pcom é a potência do último estágio completo ; t é tempo realizado na potência em que o estágio não foi completo; $\Delta \mathrm{P}=16,6 \mathrm{~W}$.

\section{Análise do lactato sanguíneo}

As amostras de sangue foram coletadas do lóbulo da orelha, sem hiperemia, em capilar heparinizado e calibrado, as quais foram imediatamente transferidas para tubos contendo $50 \mu \mathrm{l}$ de solução hemolisante (NaF 1\%). O lactato sanguíneo foi determinado em analisador eletroquímico (YSL 2300 STAT - Yellow Spring Co., EUA).

\section{Composição corporal}

A gordura corporal foi determinada por meio da medida das dobras cutâneas: triciptal, supra-ilíaca e abdominal, utilizando um compasso de dobras cutâneas (Lafayette, Calipers), segundo o protocolo proposto por Guedes (8).

\section{Análise estatística}

Os dados estão expressos com média \pm desvio padrão (DP). O teste $\mathrm{t}$ de Student foi empregado para comparar a FC correspondente ao LAn no ergômetro de braço e na piscina. O teste de correlação de Pearson foi utilizado para verificar a correlação entre as variáveis obtidas no ergômetro de braço (Pmax e LAn) com a velocidade do LAn e o menor tempo na distância de 400 m, determinados na piscina. Em todos os testes foi adotado um nível de significância de $\mathrm{p} \leq 0,05$.

\section{RESULTADOS}

A Tabela 2 apresenta os valores médios da carga (W e W/kg) e da FC referentes ao LAn determinados no ergômetro de braço e na piscina. A FC (bpm) referente ao LAn não foi estatisticamente diferente entre o ergômetro de braço $(165,0 \pm 20,8)$ e na piscina $(162,0 \pm 8,9)$. 
Tabela 2 - Valores médios + DP da intensidade e da frequência cardíaca (FC) correspondente ao limiar anaeróbio ( $L A n$ ) determinado no ergômetro de braço $(\varepsilon B)$ e na piscina $(P)(N=16)$.

\begin{tabular}{lcccc} 
& EB & \multicolumn{3}{c}{$P$} \\
\hline & $\begin{array}{c}\text { LAn } \\
(\mathrm{W} / \mathrm{kg})\end{array}$ & $\begin{array}{c}\text { FC } \\
(\mathrm{bpm})\end{array}$ & $\begin{array}{c}\text { LAn } \\
(\mathrm{m} / \mathrm{s})\end{array}$ & $\begin{array}{c}\text { FC } \\
(\mathrm{bpm})\end{array}$ \\
\hline Média & 2,16 & 165,0 & 1,14 & 162,0 \\
DP & 0,30 & 20,8 & 0,11 & 8,9
\end{tabular}

Os coeficientes de correlação entre a Pmax e a potência correspondente ao LAn determinados no ergômetro de braço com a velocidade correspondente ao LAn e o tempo de $400 \mathrm{~m}$ durante o teste na piscina estão expressos na Tabela 3. As Pmax expressas em $\mathrm{W}$ e $\mathrm{W} / \mathrm{kg}$, não foram significantemente correlacionadas com a velocidade do LAn $(r=0,37$ e $r=0,29$ ) sendo entretanto significantemente correlacionadas com o melhor tempo para nadar $400 \mathrm{~m}$ (r $=-0,66$ e $r=-0,57)$. Por outro lado, as potências correspondentes ao LAn expressas em $\mathrm{W}$ e $\mathrm{W} / \mathrm{kg}$, foram significantemente correlacionadas com a velocidade do LAn ( $\mathrm{r}=0,75$ e $\mathrm{r}=0,60)$ e o melhor tempo para nadar $400 \mathrm{~m}(\mathrm{r}=-0,74$ e $\mathrm{r}=-0,62)$, respectivamente (Figuras 1 e 2 ). A velocidade correspondente ao LAn foi significantemente correlacionada com o melhor tempo para nadar $400 \mathrm{~m}$ (r $=-0,90$ ) (Figura 3).

Tabela 3 - Coeficientes de correlação entre as variáveis determinadas no ergômetro de braço $(E B)$ e na piscina $(P)(N=16)$.

\begin{tabular}{lccccc} 
& $\begin{array}{c}\text { Pmax-EB } \\
\end{array}$ & Pmax-EB & LAn-EB & LAn-EB & LAn-P \\
& $(\mathrm{w})$ & {$[\mathrm{W} / \mathrm{Kg})$} & $(\mathrm{W})$ & {$[\mathrm{W} / \mathrm{Kg})$} & {$[\mathrm{m} / \mathrm{s})$} \\
\hline $\mathrm{T} 400 \mathrm{~m}(\mathrm{~s})$ & $-0,66^{*}$ & $-0,57^{*}$ & $-0,74^{*}$ & $-0,62^{*}$ & $-0,90^{*}$ \\
$\mathrm{LAn}-\mathrm{P}(\mathrm{m} / \mathrm{s})$ & 0,37 & 0,29 & $0,75^{*}$ & $0,60^{*}$ & $1,00^{*}$
\end{tabular}

Pmax - potência máxima; LAn - limiar anaeróbio; 4000 m - melhor tempo de 400 m nado crawl

${ }^{*} p<0,05$

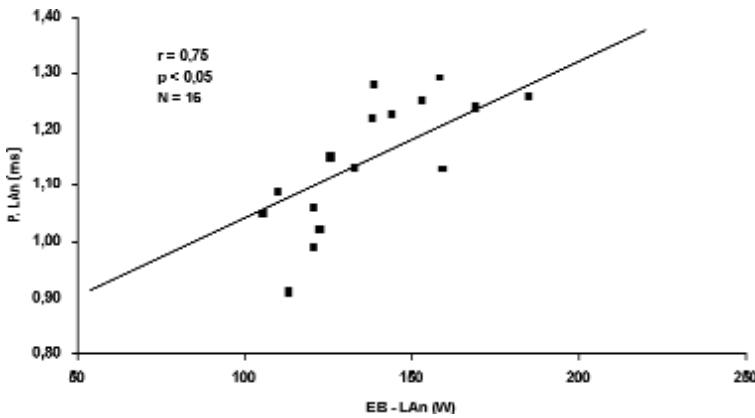

Figura 1 - Correlação entre a intensidade correspondente ao limiar anaeróbio ( $L A n$ ) determinado no ergômetro do braço $[\varepsilon B]$ e na piscina $(P]$.

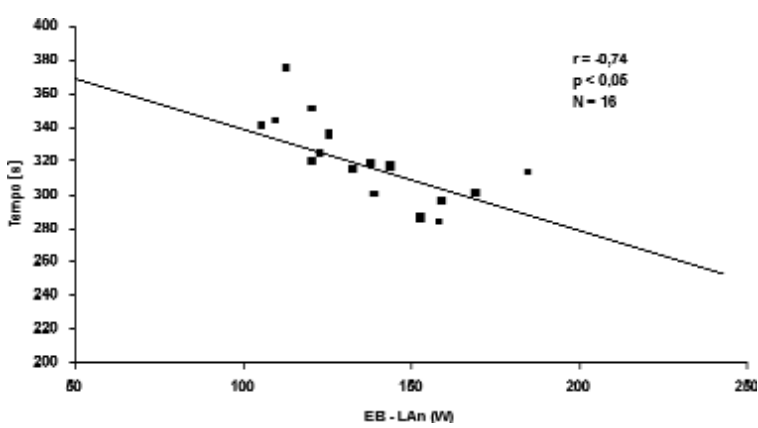

Figura 2 - Correlação entre a intensidade correspondente ao limiar anaeróbio [ $L A n$ ] determinado no ergômetro do braço $[\mathcal{E} B$ ] e o melhor tempo para nadar os 400 m estilo crawl.

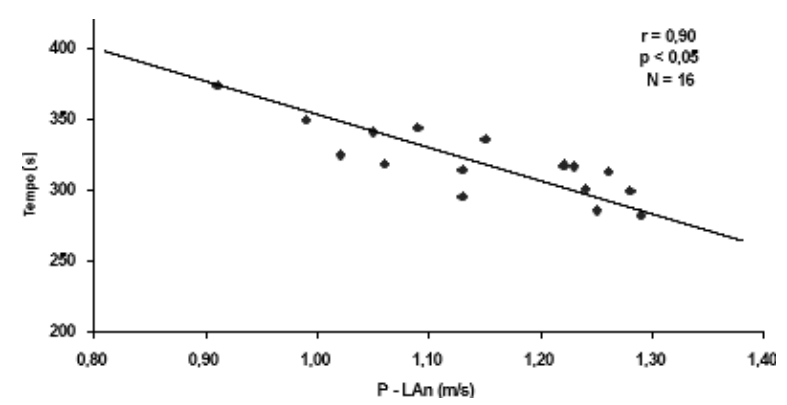

Figura 3 - Correlação entre a velocidade correspondente ao limiar anaeróbio [LAn] determinada na piscina (P) e o melhor tempo para nadar os $400 \mathrm{~m}$ estilo crawl. 


\section{DISCUSSÃO}

O objetivo deste estudo foi analisar a validade da intensidade correspondente ao LAn e a Pmax determinados em um ergômetro de braço específico, para predizer a performance na prova de $400 \mathrm{~m}$ nado crawl e a intensidade do LAn determinado na piscina. O principal achado foi que um índice submáximo determinado pela resposta do lactato ao exercício (LAn) pode apresentar maior validade do que os valores máximos (Pmax) obtidos no ergômetro de braço, para predizer a performance durante a prova de $400 \mathrm{~m}$ nado $\mathrm{crawl}$. Além disso, semelhante ao encontrado em outros estudos $(27,28)$, a intensidade correspondente ao LAn determinada na piscina, apresentou uma alta correlação com o tempo de 400 $\mathrm{m}$, explicando $81 \%$ da variação da performance nesta distância.

A determinação do $\mathrm{VO}_{2}$ max no ergômetro de braço com a finalidade de caracterizar o perfil fisiológico de triatletas $(1,22)$ e realizar a predição de performance aeróbia de nadadores tem sido bastante empregada (21). Em relação a validade para predição de performance, os dados ainda são antagônicos.

Obert et al. (21) encontraram correlação significante entre o $\mathrm{VO}_{2} \mathrm{max}(\mathrm{ml} / \mathrm{kg} / \mathrm{min})$, obtido durante o teste realizado no ergômetro de braço, e a performance nas provas de 100, 200 e 400m nado $\mathrm{crawl}$, indicando assim que este índice fisiológico é adequado para predizer a performance de nado, apesar do exercício ter sido realizado no ergômetro de braço.

Entretanto, Loftin et al. (17) não observaram correlação significante entre o $\mathrm{VO}_{2}$ max obtido no ergômetro de braço e a performance de nado $(1600 \mathrm{~m})$ em 14 triatletas recreacionais, embora para as outras modalidades (42,3 $\mathrm{km}$ de ciclismo e $10 \mathrm{~km}$ de corrida), o $\mathrm{VO}_{2}$ max determinado no ergômetro específico tenha sido correlacionado com a performance. A ausência de validade para a predição de performance aeróbia nestas condições pode não ser apenas pela falta de especificidade do ergômetro de braço, pois mesmo o $\mathrm{VO}_{2}$ max determinado no swimming flume (27), no tethered swimming $(14)$ ou na piscina $(5,28)$ também não é um bom preditor da velocidade de $400 \mathrm{~m}$ na natação.

Outro índice que tem sido muito utilizado para a predição de performance aeróbia é a intensidade (velocidade ou potência) associada ao $\mathrm{VO}_{2} \max$
$\left(\mathrm{IVO}_{2} \mathrm{max}\right)$. Muitos estudos têm verificado, em diferentes tipos de exercício (corrida e ciclismo), que a $\mathrm{IVO}_{2}$ max pode predizer melhor a performance aeróbia do que o $\mathrm{VO}_{2} \max (15,16)$, pois este índice é o que melhor reflete a integração da potência aeróbia $\left(\mathrm{VO}_{2} \mathrm{max}\right)$ e a economia de movimento (7). Para o ergômetro de braço, não foram encontrados estudos que tenham determinado diretamente a $\mathrm{IVO}_{2} \mathrm{max}$, relacionando-a com a performance na natação.

Entretanto, Hawley et al. (11) encontraram correlação significante $(r=0,70)$ entre a Pmax (maior carga atingida durante o teste incremental) determinada no ergômetro de braço e a performance na prova de $400 \mathrm{~m}$. Em nosso estudo estes dados foram reproduzidos, pois a $\mathrm{Pmax}(\mathrm{W}$ e $\mathrm{W} / \mathrm{kg}$ ) foi significantemente correlacionada com o tempo para nadar $400 \mathrm{~m}$. Estes dados devem ser analisados com cuidado, pois a Pmax atingida durante um teste incremental pode ser dependente do protocolo empregado (taxa de incremento e duração do estágio), sem que se modifique necessariamente $o$ $\mathrm{VO}_{2} \max (10,25)$.

A resposta do lactato sanguíneo ao exercício, identificado por diferentes terminologias (limiar de lactato, limiar anaeróbio, início do acúmulo de lactato no sangue, máxima fase estável de lactato), tem mostrado maior validade para a predição da performance aeróbia do que o $\mathrm{VO}_{2} \max (6,29)$. A utilização de uma concentração fixa de lactato $(4 \mathrm{mM})$ para a identificação máxima fase estável de lactato (MLACSS) pode não ser apropriada, pois o estado nutricional (18), a variabilidade individual (23) e o tipo de exercício (3), podem influenciar nesta predição. Entretanto, diversos estudos têm mostrado que a intensidade correspondente a $4 \mathrm{mM}$ de lactato sanguíneo, é válida para a predição de performance aeróbia na natação $(27,28)$. Em nosso estudo foi observada uma alta correlação $(r=-0,90)$ entre a velocidade equivalente ao LAn no teste de piscina com a performance na prova de $400 \mathrm{~m}$ crawl, confirmando assim que o uso de valores fixos para a identificação da resposta do lactato sanguíneo é válido para a predição de performance, mesmo em um grupo relativamente homogêneo em termos do melhor tempo para os $400 \mathrm{~m}$ crawl (CV $=8 \%$ ).

Em relação ao ergômetro de braço, foi encontrada também uma correlação significante $(r=-0,74)$ 
entre o LAn e o tempo nos $400 \mathrm{~m}$, sugerindo que a ausência de validade do $\mathrm{VO}_{2} \max$ determinado no ergômetro de braço para a predição de performance é mais dependente da menor capacidade de predição do índice $\left(\mathrm{VO}_{2} \max \right)$ do que da falta de especificidade do ergômetro. É importante destacar, entretanto, que a correlação encontrada é apenas moderada, explicando bem menos a variação de performance (54\%) do que a encontrada pelo LAn determinado na piscina. Na natação, a performance pode não depender apenas dos aspectos metabólicos, mas também da técnica de nado e da densidade corporal, não podendo estes últimos serem avaliados durante o exercício realizado no ergômetro de braço.

A FC correspondente ao LAn não foi diferente entre o ergômetro de braço e piscina, indicando que a relação entre a FC e a resposta metabólica é similar, embora a massa muscular empregada e a posição do corpo sejam diferentes. Isto poderia apresentar algumas implicações práticas interessantes, como a transferência dos dados obtidos em laboratório para o controle da intensidade de treinamento na piscina.

Entretanto, a maior variabilidade da FC no ergômetro de braço (indicado pelo maior DP), sugere que esta informação tenha que ser utilizada com ressalvas. Com base nos resultados encontrados nas condições experimentais deste estudo, pode-se concluir que a validade do ergômetro de braço para a avaliação aeróbia de nadadores, é maior quando é utilizada a resposta do lactato sanguíneo (LAn) do que os valores Pmax obtidos durante um teste incremental.

\section{CORRESPONDÊNCIA}

\section{B.S. Denadai}

Laboratório de Avaliação da Performance Humana IB, UNESP

Av. 24 A, 1515

Bela Vista - Rio Claro - SP

CEP - 13506-900

Brasil

bdenadai@rc.unesp.br 


\section{REFERÊNCIAS BIBLIOGRÁFICAS}

1. Albrecht TJ, Foster VL, Dickinson AL, Debever JM (1986).

Triathletes: exercise parameters measured during bicycle, swim bench, and treadmill testing. Med Sci Sports Exerc 18: 86

2. Bar-Or O, Zwiren LD (1975). Maximal oxygen consumption test during arm exercise - reliability and validity. J Appl Physiol 38: 424-426

3. Beneke R, Von Duvillard SP (1996). Determination of maximal lactate steady state response in selected sports events. Med Sci Sports Exerc 28: 241-246

4. Costill DL, Thomas R, Hargreaves M (1985a). Effects of reduced training on muscular power in swimmers. Phys Sports Med 13: 94-101

5. Costill DL, Kovaleski J, Porter D, Kirwan J, Fielding R, King D (1985b). Energy Expenditure during front crawl swimming: Predicting success in middle distance events. Int J Sports Med 6: 266-270

6. Coyle EF (1995). Integration of the physiological factors determining endurance performance ability. In: Holloszy JO (ed). Exercise and Sport Sciences Reviews. Baltimore, Williams \& Wilkins, 25-63

7. Daniels JA, Daniels N (1992). Running economy of elite male and elite female runners. Med Sci Sports Exerc 24: 483-489 8. Guedes DP (1984). Composição corporal: princípios, técnicas e aplicações. Londrina: Associação dos Professores de Educação Física.

9. Guglielmo LGA, Denadai BS (2000). Assessment of anaerobic power of swimmers: the correlation of laboratory tests on an arm ergometer with field tests in a swimming pool. $J$ Strength Cond Res 14: 395-398

10. Hansen JE, Casaburi R, Cooper DM, Wasserman K (1988). Oxygen uptake as related to work rate increment during cycle ergometer exercise. Eur J Appl Physiol 57: 140-145

11. Hawley JA, Williams MM, Vickovic MM, Handcock PJ

(1992). Muscle power predicts freestyle swimming performance. Br J Sports Med 26: 151-155

12. Hawley JA, Williams MM (1991). Relationship between upper body anaerobic power and freestyle swimming performance. Int J Sports Med 12: 1-5

13. Heck H, Mader A, Hess G, Mucke S, Muller R, Hollmann $\mathrm{W}$ (1985). Justification of the $4 \mathrm{mmol} / 1$ lactate threshold. Int $J$ Sports Med 6: 117-130

14. Kohrt WM, Morgan DW, Bates B, Skinner JS (1987). Physiological responses of triathetes to maximal swimming, cycling, and running. Med Sci Sports Exerc 19: 51-55

15. Lacour JR, Padilla-Magunacelaya S, Barthelemy JC, Dormois D (1990). The energetics of middle-distance running. Eur J Appl Physiol 60: 38-43

16. Lindsay FH, Hawley JA, Myburgh KH, Schomer HH,

Noakes TD, Dennis SC (1996). Improved athletic performance in highly trained cyclists after interval training. Med Sci Sports Exerc 28: 1427-1434

17. Loftin M, Warren BL, Zingraf S, Brandon JE, Skudlt A, Scully B (1988). Peak Physiological function and performance of recreational triathletes. J Sports Med Phys Fitn 28: 330-335 18. Maassen N, Buse MW (1989). The relationship between lactic acid and work load--a measure for endurance capacity or an indicator of carbohydrate deficiency? Eur J Appl Physiol 58: 728-737

19. Mader A, Madsen O, Holtmann W (1980). Zur beurteilung der laktaziden energiebereitstellung fur trainings und wett- kampf-leistungen im sportstschwimmen. Leistungsport 10: 163169

20. Magel JR, Foglia GF, McArdle WD, Gutin B, Pechar GS, Katch FI (1975). Specificity of swim training on maximal oxygen uptake. J Appl Physiol 38: 151-155

21. Obert P, Falgairette G, Bedu M, Coudert J (1992).

Bioenergetic characteristics of swimmers determined during an arm-ergometer test and during swimming. Int J Sports Med 13: 298-303

22. O'toole ML, Hiller DB, Crosby LO, Douglas PS (1987). The ultraendurance triathlete: a physiological profile. Med Sci Sports Exerc 19: 45-50

23. Stegmann H, Kindermann W, Schnabel A (1981). Lactate kinetics and individual anaerobic threshold. Int J Sports Med 2: $160-165$

24. Sawka NM (1986). The physiology of upper body exercise. Exercise and Sports Science Reviews 14: 175-211

25. Sawka MN, Foley ME, Pimental NA, Toner MM, Pandolf KB (1983). Determination of maximal aerobic power during upper-body exercise. J Appl Physiol 54: 113-117

26. Vokac Z, Bell H, Bautz-Holter E, Rodahl K (1975). Oxygen uptake/heart rate relationship in leg and arm exercise, sitting and standing. J Appl Physiol 39: 54-59

27. Wakayoshi K, Yoshida, T, Udo M, Kasai T, Moritani, T, Mutoh Y, Miyashita M (1992). A simple method for determining critical speed as swimming fatigue threshold in competitive swimming. Int J Sports Med 13: 367-371

28. Wakayoshi K, Yoshida T, Udo M, Harada T, Moritani T (1993). Does critical swimming velocity represent exercise intensity at maximal lactate steady-state? Eur J Appl Physiol 66: 90-95

29. Weltman A. (1995) The blood lactate response to exercise. USA: Human kinetics. 